\title{
Depolarized Rayleigh Scattering and Stereoregularity of Polystyrene Chains.* II. Interpretation
}

\author{
Georges Fourche and Bernard Lemaire \\ Centre de Recherches Paul Pascal, Domaine Universitaire \\ 33, Talence, France.
}

(Received March 27, 1972)

\begin{abstract}
The molecular optical anisotropy of isotactic and atactic polystyrene, and their oligomers has been interpreted according to the rotational isomeric state model. The results obtained allowed us to determine the statistical weights of mesoand racemic 2,4-diphenylpentane and the respective orientation of the phenyl rings in these molecules. Analysis also showed that isotactic polystyrene in solution had the same local conformation as the meso pentane. This conformation consisted of a succession of very long helical segments of type $3_{1}$. In the case of atactic polystyrene a conformation of identical nature was found, though the length of isotactic sequences was much smaller.

Finally, this study shows that it has been possible to interpret, by means of conformational parameters of model compounds, all the experimental values observed for isotactic and atactic polymers, thus pointing out the utility of depolarized Rayleigh Scattering as a method for the determination of the average conformation of polymers as well as that of short chains.
\end{abstract}

KEY WORDS Optical Anisotropy / Rayleigh Scattering / Stereoregularity / Local Conformation / Rotational Isomerism / Polystyrene / Diphenylpentane /

For several years studies of calculating the average molecular optical anisotropy $\left\langle\gamma^{2}\right\rangle$ of flexible chains have realized an important development in connection with the very interesting and sometimes unique information obtained in certain experimental studies. ${ }^{1-3}$ Thus Jernigan and Flory, ${ }^{4}$ Nagai, ${ }^{5}$ and other authors ${ }^{6,7}$ have presented, independently, various treatments on this molecular quantity for relatively simple polymers such as polyethylene and poly(oxyethylene). Very recently an extension of these methods to the study of molecular optical anisotropy of vinyl polymers has been carried out by Tonelli, Abe, and Flory. ${ }^{8}$ In the present work, we shall use the $\left\langle\gamma^{2}\right\rangle$ formulation given by these last three authors and shall consequently limit the theoretical presentation to its essentials for this study.

* Part of this work was presented (G-F) at the 9th microsimposium on "Thermodynamics of Interactions in Polymer Solutions,"' Prague, Sept. 6-9, 1971.

\section{AVERAGE MOLECULAR OPTICAL ANISOTRORY}

For the convenience of our presentation, we have pictured in Figure 1 a portion of atactic vinyl chain in its planar trans conformation. We designate by $\mathrm{d}$ each asymmetric carbon atom linked to an $\mathrm{R}$ group placed in front of the skeletal plane of the molecule and by 1 that for which $\mathbf{R}$ is situated behind this same plane.

Following this notation it will be possible to represent an isotactic chain by a dddd...or $1111 .$. succession, a syndiotactic chain by a dldldl... or ldldld ... arrangement, and an atactic chain by a dlldldd... arrangement.

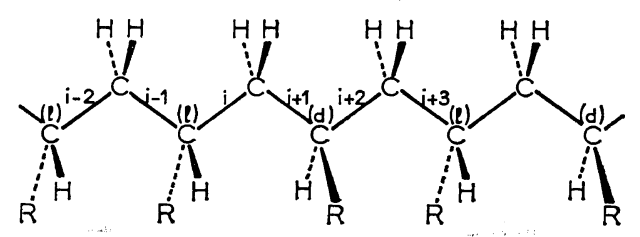

Figure 1. 
The conformation of a vinyl polymer with the general formula $\mathrm{H}-\left(\mathrm{CH}_{2}-\mathrm{CHR}\right)_{n / 2}-\mathrm{CH}_{3}\left(\mathrm{R}=\mathrm{C}_{6} \mathrm{H}_{5}\right.$ in our case), where $n$ is the number of skeletal bonds, can be described by meso $\mathrm{dd}$ or 11 and racemic $\mathrm{dl}$ or $\mathrm{ld}$ sequences of $-\mathrm{CHR}-\mathrm{CH}_{2}-\mathrm{CHR}-$ dyads. The conformations generated by internal rotations around the various bonds of the chain are correctly treated in terms of discrete rotational states, generally chosen to coincide with the minima of the internal rotation potential function. It is assumed that for aliphatic $\mathrm{C}-\mathrm{C}$ bonds these minima are represented by trans $(t)$, gauche $(g)$, and gauche prime $\left(g^{\prime}\right)$ states, which nearly correspond to the internal rotation positions $\varphi=0,+120$, and $-120^{\circ}$, respectively.

Partition Function and Statistical Weight Matrices

It can be shown that the conformation of the chain as a whole is entirely specified if the meso and racemic character of $-\mathrm{CHR}-\mathrm{CH}_{2}-\mathrm{CHR}-$ dyads is known. Under these conditions, the partition function of the chain is given by

$$
Z=\mathbf{J}^{*}\left[\prod_{k=1}^{x-1} \mathbf{U}_{k}^{(2)}\right] \mathbf{J}
$$

where $x=n / 2$ is the degree of polymerization of the chain, and $\mathbf{J}^{*}$ and $\mathbf{J}$ are respectively the row and column vectors defined elsewhere ${ }^{9}$ and are of the order corresponding to the number (here three) of internal rotation tates chosen to describe the eligible conformations of each bond of the molecule. The statistical weight matrix relative to the $K$-th dyad is defined by

$$
\mathbf{U}_{k}{ }^{(2)}=\left(\mathbf{U}_{\mathrm{a}}{ }^{\prime} \mathbf{U}_{\mathrm{ab}}^{\prime \prime}\right)_{k}
$$

where the $d$ or 1 character of the asymmetric center of the dyad in question is noted by a and $b$.

Considering the triperiodicity of the internal rotation potential chosen for each bond of the molecule, the corresponding statistical weight are of the order $3 \times 3$. For the two bonds of a dyad dd, we have

$$
\begin{aligned}
\mathbf{U}_{\mathrm{d}}^{\prime} & =\left|\begin{array}{llc}
\eta & 1 & \tau \\
\eta & 1 & \tau \omega \\
\eta & \omega & \tau
\end{array}\right| \\
\mathbf{U}_{\mathrm{dd}}^{\prime \prime} & =\left|\begin{array}{ccc}
\eta \omega^{\prime \prime} & \tau \omega^{\prime} & 1 \\
\eta & \tau \omega^{\prime} & \omega \\
\eta \omega^{\prime} & \tau \omega \omega^{\prime \prime} & \omega^{\prime}
\end{array}\right|
\end{aligned}
$$

For a dyad 11, the statistical weight matrices are given in an analogous manner by

$$
\begin{aligned}
\mathbf{U}_{1}^{\prime} & =\left|\begin{array}{lll}
\eta & \tau & 1 \\
\eta & \tau & \omega \\
\eta & \tau \omega & 1
\end{array}\right| \\
\mathbf{U}_{11}^{\prime \prime} & =\left|\begin{array}{ccc}
\eta \omega^{\prime \prime} & 1 & \tau \omega^{\prime} \\
\eta \omega^{\prime} & \omega^{\prime} & \tau \omega \omega^{\prime \prime} \\
\eta & \omega & \tau \omega^{\prime}
\end{array}\right|
\end{aligned}
$$

The statistical weight matrices of type $\mathbf{U}^{\prime}$ are applicable to those bonds such as $i$, which links CHR to $\mathrm{CH}_{2}$, and the matrices of type $\mathbf{U}^{\prime \prime}$ to bonds which, like $i+1$, connects $\mathrm{CH}_{2}$ to CHR.

In the case of a syndiotactic disposition $\mathrm{dl}$ or ld of the asymmetric carbon pair joined to the $i$ and $i+1$ bonds, we have

$$
\begin{aligned}
& \mathbf{U}_{\mathrm{d} 1}^{\prime \prime}=\left|\begin{array}{ccc}
\eta & \omega^{\prime} & \tau \omega^{\prime \prime} \\
\eta \omega^{\prime} & 1 & \tau \omega \\
\eta \omega^{\prime \prime} & \omega & \tau \omega^{\prime 2}
\end{array}\right| \\
& \mathbf{U}_{1 \mathrm{~d}}^{\prime \prime}=\left|\begin{array}{ccc}
\eta & \tau \omega^{\prime \prime} & \omega^{\prime} \\
\eta \omega^{\prime \prime} & \tau \omega^{\prime 2} & \omega \\
\eta \omega^{\prime} & \tau \omega & 1
\end{array}\right|
\end{aligned}
$$

The $\mathbf{U}^{\prime}$ matrices to be used in connection with $\mathbf{U}_{\mathrm{d} 1}^{\prime \prime}$ and $\mathbf{U}_{1 \mathrm{~d}}^{\prime \prime}$ are those given by eq 3 and 5 respectively.

The statistical weights in the matrices described above take into account both the "first order" and 'second order"' interactions, the former being only a function of a single rotational angle, and the latter of two internal rotation angles.

The begin with, let us consider the case, where the interactions under consideration involve the following pairs of atom groups: $\mathrm{CH}_{2}, \mathrm{CH}$, and $R$, separated by three skeletal bonds of the molecule.

We have represented, in Figure 2 below, the internal rotation states $t, g$, and $g^{\prime}$, around the $\mathrm{CHR}-\mathrm{CH}_{2}$ bond. One of the ends of the chain having been arbitrarily selected, the conformations will be generated by going through the molecule from left to right, the internal rotational being positive in clockwise. We consider in the course of this study that the $\mathrm{CH}_{2}$ and $\mathrm{CH}$ groups have approximately equivalent effects, that is, the interactions created by these two groups are of the same order of magnitude. On this basis, a factor $\tau$ will be chosen to re- 


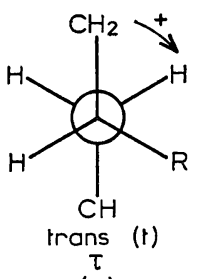

(†)

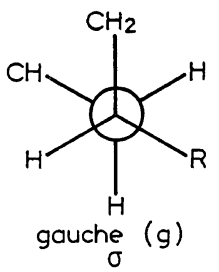

(1)

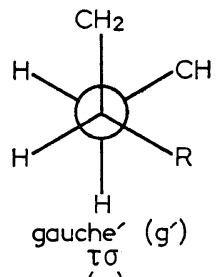

$(\tau)$
Figure 2.

present the interactions of a $\mathrm{CH}$ group with an $\mathrm{R}$ group in the trans conformation. A factor $\sigma$ will be assigned to the gauche conformation involving interactions between $\mathrm{CH}_{2}$ and $\mathrm{CH}$ of the same nature as those observed in normal alkanes. Lastly, the gauche prime conformation places a $\mathrm{CH}$ at equal distance between $\mathrm{CH}_{2}$ and $\mathrm{R}$ groups and will be characterized by a statistical weight $\sigma \tau$. For greater convenience in discussion and expression of the formulae we can normalize these diverse factors to a weight of unity for the gauche state. Under these conditions we will obtain, $\eta, 1$, and $\tau$ for the statistical weights relative to positions $t, g$, and $g^{\prime}$ (with $\eta=\tau / \sigma$ ). Thus, with this new formulation, the parameter $\eta$ is introduced for each conformation in which the $\mathrm{R}$ substituent is gauche with respect to a $\mathrm{CH}$ group, from which it is separated by three skeletal bonds. This statistical weight is assigned relative to a value of unity for a $\mathrm{CH}_{2}$ group situated in an equivalent manner with regard to the $\mathrm{CH}$ groups. Finally, the factor $\tau$ is involved in conformations in which the ensemble of $\mathrm{CH}_{2}$ and $\mathrm{R}$ groups is gauche compared to $\mathrm{CH}$.

The factors $\omega, \omega^{\prime}$, and $\omega^{\prime \prime}$ still remain to be defined. These are related to "second order" interactions involving atom groups separated by four skeletal bonds and consequently dependent on internal rotation angles around two consecutive bonds. The statistical weight $\omega$ characterizes the interactions between pairs of atom groups $\mathrm{CH}_{2}$ or $\mathrm{CH}, \omega^{\prime}$ is related to those between $\mathrm{CH}_{2}$ and $\mathrm{R}$, and $\omega^{\prime \prime}$ is connected to interactions between two $\mathbf{R}$ groups. All these interactions are very strong and the corresponding conformations are extremely unfavorable. Taking into account the van der Waals radius of the methyl group $(2.0 \AA)$ we can note that the $\mathrm{CH}_{2} \cdots \mathrm{CH}_{2}$ or $\mathrm{CH} \cdots \mathrm{CH}$ interaction of weight $\omega$ places the interacting groups $2.69 \AA$ apart and that severe repulsions must appear. In normal alkanes a value $\omega=0.041$ at $25^{\circ} \mathrm{C}$ has been determined $^{6}$ for this same type of interaction. In like manner, the $\mathrm{R} \cdot \mathrm{CH}_{2}$ interaction of weight $\omega^{\prime}$ places the two nearest carbon atoms about $2.60 \AA$ apart, that is, at a distance shorter by $1 \AA$ than the sum of their Van der Waals radii (about $1.8 \AA$ ). Important steric overlaps must therefore occur. Finally, the phenyl groups being relatively bulky groups, we can conclude for the same reasons as above that the conformations which generate $R \cdot . R$ interactions between these groups are sterically unacceptable. These considerations lead us to put $\omega=\omega^{\prime}=\omega^{\prime \prime}$ $=0$, due to the fact that the corresponding interaction energies are all equal to or greater than $2000 \mathrm{cal} \mathrm{mol}^{-1}$. These statistical weights are represented by Boltzman factors of the form $\exp (-\Delta E / R T)$ where $\Delta E$ is the difference between the energies of internal rotation for the considered conformation and the reference trans state, $R$ the gas constant, and $T$ the temperature of the experiment.

\section{Optical Anisotropy}

The statistical mechanical average value of the molecular optical anisotropy of PS chains can be written in the following form

$$
\left\langle\gamma^{2}\right\rangle=2 Z^{-1} \mathscr{J}^{*} \mathscr{P}_{1}\left[\prod_{k=1}^{x-1} \mathscr{P}_{k}^{(2)}\right] \mathscr{P}_{n} \mathscr{P}_{n+1} \mathscr{J}
$$

where $Z$ is the partition function defined by eq $1, \mathscr{J}^{*}$ and $\mathscr{J}$ are row and column vectors ${ }^{9}$ of the order 33 and $\mathscr{P}_{k}{ }^{(2)}$ a square matrix of the corresponding order and relative to the $K$-th dyad. We have obviously

$$
\mathscr{P}_{k}^{(2)}=\left(\mathscr{P}_{\mathrm{a}}{ }^{\prime} \mathscr{P}_{\mathrm{ab}}^{\prime \prime}\right)_{k}
$$

the matrices $\mathscr{P}_{\mathrm{a}}^{\prime}$ and $\mathscr{P}_{\mathrm{ab}}^{\prime \prime}$ being constructed in particular with the help of the statistical weight matrices $\mathbf{U}_{\mathrm{a}}^{\prime}$ and $\mathbf{U}_{\mathrm{ab}}^{\prime \prime}$ previously described.

In the case of an isotactic chain, we have $a=b=d$ (or 1 ) and for a syndiotactic chain $a=d$ and $b=1$ (or $a=1$ and $b=d$ ).

The explicit form of $\mathscr{P}^{\prime}$ and $\mathscr{P}^{\prime \prime}$ matrices, relative to $\mathrm{CHR}-\mathrm{CH}_{2}$ bonds of type $i$ and $\mathrm{CH}_{2}-$ CHR bonds of type $i+1$, respectively is given by the following expression 


$$
\mathscr{P}_{i}=\left|\begin{array}{ccc}
\mathbf{U} & \left(\mathbf{U} \times \boldsymbol{\gamma}^{\mathrm{R}}\right)(\|\mathbf{T} \times \mathbf{T}\|) & 3 / 4\left(\boldsymbol{\gamma}^{\mathrm{R}} \cdot \boldsymbol{\gamma}^{\mathrm{C}} \cdot \mathbf{U}\right) \\
\mathbf{0} & \left(\mathbf{U} \times \mathbf{E}_{9}\right)(\|\mathbf{T} \times \mathbf{T}\|) & 3 / 2\left(\mathbf{U} \times \boldsymbol{\gamma}^{\mathrm{C}}\right) \\
\mathbf{0} & \mathbf{0} & \mathbf{U}
\end{array}\right|_{i}
$$

In this equation $\boldsymbol{\gamma}_{i}{ }^{\mathrm{R}}$ and $\boldsymbol{\gamma}_{i}{ }^{\mathrm{C}}$ represent the row and column forms of the optical anisotropy tensor, ${ }^{3,9} \boldsymbol{\gamma}_{i}=\boldsymbol{\alpha}_{i}-\bar{\alpha}_{i} \mathbf{E}_{3}$ associated with the bond of group $i$ while $\boldsymbol{\alpha}_{i}$ is the polarizability tensor for the bond $i, \bar{\alpha}_{i}=1 / 3$ trace $\left(\boldsymbol{\alpha}_{i}\right)$ is its mean value, and $\mathbf{E}_{3}$ the unit matrix of order 3 . The $\|\mathbf{T} \times \mathbf{T}\|_{i}$ matrices defined elsewhere ${ }^{9}$ are functions of the internal rotation angle $\varphi_{i}$ associated with the corresponding skeletal bond and of the supplement $\theta_{i}$ of the valence angle formed by the pair of bonds $i$ and $i+1$. In the case of PS, the angles $\theta$ remain identical and consequently the index $i$ could be omitted. Lastly, $\mathbf{E}_{9}$ is the unit matrix of order 9 and $\mathbf{U}_{i}$ the statistical weight matrix involved in the internal rotation around the corresponding bond. From eq 11 we can see that the product $\mathscr{P}^{\prime} \mathscr{P}^{\prime \prime}$ can also be written as $\mathscr{P}^{\prime}\left(\mathbf{U}^{\prime}, \mathbf{T}, \boldsymbol{\gamma}^{\prime}\right) \mathscr{P}^{\prime \prime}\left(\mathbf{U}^{\prime \prime}, \mathbf{T}, \boldsymbol{\gamma}^{\prime \prime}\right)$. The optical anisotropy tensors $\boldsymbol{\gamma}^{\prime}$ and $\boldsymbol{\gamma}^{\prime \prime}$ are related to atom groups $\mathrm{CHR}-\mathrm{C}$ and $\mathrm{CH}_{2}-\mathrm{C}$, respectively, which are identically reproduced along the chain. We shall not give here the rather complex explicit form ${ }^{9,17}$ of these tensors because it is not necessary for our interpretation. Let us simply remark that in the construction of tensor $\gamma^{\prime}$ the rotational angle $\chi$ of the phenyl ring around the $\mathrm{C}-\mathrm{R}$ bond must be known. According to our conventions, this angle will be positive (see Figure 3 ) when the rotation is made clockwise.

The very likely case ${ }^{10,11}$ where the different phenyl rings perform torsional vibrations of small magnitude around their equilibrium position $\chi=0$ can be taken into account in the evaluations of $\boldsymbol{\gamma}^{\prime}$ by averaging the diverse trigonometric lines (functions of $\chi$ ) which are contained therein.

Up to now we have only considered the case of perfectly stereoregular chains of the isotactic or syndiotactic type. However, if the configuration $\mathrm{d}$ or 1 of the asymmetric centers does not occur as a regular succession along the chain, that is to say if some stereoirregular units randomly appear in the molecule, a more elaborate treatment should be used. The chain must be treated as a copolymer containing a statistical set of diverse molecular species differing in the succession of the stereochemical configuration of the monomer units. We assume that the operative conditions during the synthesis of the collections of chains make possible the definition

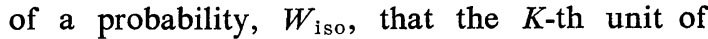
any chain has the same stereochemical configuration as the preceding one. Consequently $W_{\text {syn }}=$ $1-W_{\text {iso }}$ will be the probability of a syndiotactic placement of the monomer in question relative to its predecessor. Moreover, we shall assume that the probability $W_{\text {iso }}$ is independent of the index $K$ and that for all the molecules in the sample this probability is identical. Finally, we shall make the hypothesis that the probability $W_{\text {iso }}$ is independent of the stereochemical configuration of the preceding dyad, that is to say, the distribution of stereoirregular units is randomly produced according to Bernoulli's law. In practice we obtain a representative set of molecules, each composed of $x$ monomer units, by the random drawing of a series of $x-1$ numbers between 0 and 1 . The numbers of this series smaller than $W_{\text {iso }}$ will be taken to specify an isotactic dyad for which the $\mathrm{d}$ or 1 character of the preceding monomer unit will be conserved. On the other hand, the numbers greater than $W_{\text {iso }}$ will indicate a syndiotactic dyad. Then the products $\prod_{k=1}^{x-1} \mathscr{P}_{k}{ }^{(2)}$ and $\prod_{k=1}^{x-1} \mathbf{U}_{k}^{(2)}$ which appear in eq 9 and 1 will be calculated and the molecular optical anisotropy of this species evaluated. Next, the process will be repeated for a certain number of other species until a representative ensemble of chains in solution is obtained. Usually 15 to 25 generations are sufficient.

\section{Structural Data and Bond Optical Anisotropies}

All the numerical calculations are carried out by using the following data $^{8}$

$$
\begin{aligned}
\theta & =\pi-\widehat{\mathrm{CCC}}=68^{\circ} \\
\pi-\widehat{\mathrm{CCH}} & =\pi-\widehat{\mathrm{HCH}}=\pi-\widehat{\mathrm{CCR}} \cong 70^{\circ} 32^{\prime}
\end{aligned}
$$

together with the bond optical anisotropies (see definition in eq 2 of part I) obtained from previous experimental determinations ${ }^{6,12}$ as shown below

$$
\begin{aligned}
\gamma_{\mathrm{CC}} & =1.31 \AA^{3}, \quad \gamma_{\mathrm{CH}}=0.22 \AA^{3}, \\
\gamma_{\mathrm{C}_{6} \mathrm{H}_{6}} & =\gamma_{\varphi}=6.97 \AA^{3} .
\end{aligned}
$$




\section{STUDY OF 2,4-DIPHENYLPENTANE}

Up to now little work has been done to study the preferential conformations in solution taken by the 2,4-diphenylpentane (DPP), a dimer molecule of PS. The first two studies in this field are those by Bovey, et al., ${ }^{13,14}$ and $\mathrm{Lim}$ and coworkers $^{19}$ who have interpreted NMR spectra of optical isomers of this molecule. Later, Williams and Flory ${ }^{15}$ studied the stereochemical equilibria of PS oligomers and were able to obtain the proportions of meso and racemic forms of DPP. It must be noted here that, in all these works, the orientation of the phenyl group (R) with respect to the bonds of the carbon framework is $a$ priori supposed to be fixed, so that the plane of the aromatic ring coincides with that defined by the H-C-R pair of bonds. Very recently, however, a complete calculation of the conformational energies of these molecules, where the relative disposition of phenyl group is taken into account, has been carried out by Gorin and Monnerie $^{11}$ by means of classical functions of potential energy.

The study of molecular optical anisotropy, a property which is known to be particularly sensitive to the presence of aromatic residues, ${ }^{16}$ should make possible an accurate verification of the above theoretical predictions as well as of certain results in the literature.

In this work we thus propose to study the preferred local conformations of meso and racemic isomers of DPP and to use the information obtained on these molecules in the interpretation of conformations of isotactic and atactic PS chains, respectively. This last polymer corresponds, in fact, to a random distribution along a chain of the meso and racemic dyads which are in question in the previous section. The entire study of DPP conformations necessitates the knowledge of preferential conformations of the aliphatic backbone, the mutual orientation of aromatic rings, and the statistical weights of the different conformers. We shall try to develop successively these several points, first for the meso isomer and then for the racemic.

\section{Meso-DPP}

We have represented, for greater convenience, the meso pentane in its fully extended conformation in Figure 3, in which are shown the

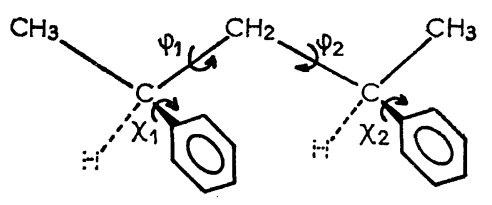

Figure 3. Meso-(dd)-diphenylpentane in its trans conformation.

internal rotation angles $\varphi_{1}$ and $\varphi_{2}$ together with the rotation angles* $\chi_{1}$ and $\chi_{2}$ of phenyl groups (R) measured relative to the $\mathbf{H}-\mathrm{C}-\mathrm{R}$ planes which bisect the valence angles of the aliphatic skeleton. For all these angles, the rotation is taken as positive when it is clockwise, as indicated before.

Due to the triperiodicity of the potential related to the internal relation around the C-C bonds of the skeleton, there exist 9 possible conformations for the four bond sequence of the optical isomer under consideration.

The critical examination in the preceding paragraph of interatomic distances and the corresponding interactions in this molecule has readily shown that for a dyad dd, only two conformations $\left(\operatorname{tg}^{\prime}\right.$ and $g t$ ) are allowed, each with a statistical weight $\eta$. For greater clarity we have drawn in Figure 4, in the case of the $\operatorname{tg}^{\prime}$ conformation, the phenyl... CH interaction corresponding to this statistical weight.

On the basis of these observations we see that the statistical weight $\eta$ and the angles $\varphi$ and $\chi$ alone remain to be determined. Each of the $t^{\prime}$ and $g t$ conformations of meso pentane have the same statistical weight, thus they are equally probable, and consequently the mean molecular

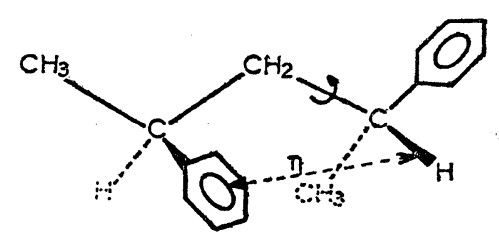

Figure 4. Meso-(dd)-diphenylpentane in its $t g^{\prime}$ conformation.

* The method of calculation of bond optical anisotropy tensors derived in ref. 17 has been conveniently modified in order to make it possible to distinguish the two internal rotation angles, $\chi_{1}$ and $\chi_{2}$, of the phenyl rings. 
optical anisotropy of this molecule does not depend explicitly on the factor $\eta$. It reduces indeed to the arithmetic average of the molecular optical anisotropies of the possible conformational isomers. Thus we can determine the value of the parameter $\chi$ by comparing the experimental and calculated optical anisotropies, the angles $\varphi$ being already known with sufficient accuracy. ${ }^{11}$ It is also possible at this stage to analyze separately the influence of a small vibration of the rings around their equilibrium position $(\chi=0)$ or of a slight alteration of the internal rotation positions $g$ or $g^{\prime}$, as suggested in some studies. ${ }^{10,11}$

At this point of our development it is interesting to make the following remarks. The mean value of the experimental optical anisotropies of meso-DPP in cyclohexane and 1,1,3trimethyl-cyclohexane solutions is $137.5 \AA^{6}$; this value is considerably larger than the experimental optical anisotropy ${ }^{18}$ of normal pentane in cyclohexane which is $3.03 \AA^{6}$. Consequently, the major contribution to the optical anisotropy of this isomer must arise from aromatic rings. In fact a simple calculation shows that the theoretical molecular optical anisotropy of two benzene molecules joined together and parallel is four times higher than that of an isolated molecule. This leads to $\gamma_{(2 \varphi \|)}^{2}=4 \gamma_{\varphi}{ }^{2}=194.4 \AA^{6}$ if we adopt the value ${ }^{12} 48.60 \AA^{6}$ for the molecular optical anisotropy of benzene in cyclohexane solution. On the contrary, if the planes of the two benzene molecules are perpendicularly oriented it is found that the theoretical anisotropy remains unchanged. Thus $\gamma_{(2 \varphi \perp)}^{2}=\gamma_{\varphi}{ }^{2}=48.60 \AA^{6}$. From these simple qualitative considerations it can be deduced that a rather strong tendency to parallelism must exist between the orientations of the phenyl rings in the $\operatorname{tg}^{\prime}$ and $g t$ conformations of meso-DPP.

We have reported in Table I theoretical molecular optical anisotropies of meso-DPP calculated (eq 9) from bonds, optical anisotropies, and structural data mentioned above and according to diverse choices of the angles $\varphi$ and $\chi$. We note that the model considered in case $I$, in which the internal rotational states are located at the usual symmetrical positions $0,+120^{\circ}$, and $-120^{\circ}$ and the angles $\chi$ have a zero value (which should correspond to the most stable positions according to elementary symmetry considerations) leads to a molecular optical anisotropy much smaller than the experimental value. We have verified that the introduction of a slight symmetrical oscillation, from 5 to $10^{\circ}$, of each one of the rings around its equilibrium position $(\chi=0)$ lowered this last results still more. Nevertheless, the effect observed always remained small. Therefore, we have been led to study successively, in cases II and III, the influence of a slight modification of $15^{\circ}$ for the internal rotation angles $g$ and $g^{\prime}$ and of a small rotation of $10^{\circ}$ of one cycle as predicted from the calculation $^{11}$ of the conformational energies of these molecules. We see that the molecular optical anisotropy regularly increases from models I to III. Let us notice here that model III corresponds to the conformation of minimum energy ${ }^{11}$ calculated by taking into account the van der Waals interactions between non bonded atoms,

Table I. Calculated values of the molecular optical anisotropy of meso-diphenylpentane for various choices of the angles $\varphi$ and $\chi$

\begin{tabular}{|c|c|c|c|c|c|c|}
\hline Models & $\varphi_{1}$ & $\varphi_{2}$ & $\chi_{1}$ & $\chi_{2}$ & Conformation & $\left\langle\gamma^{2}\right\rangle, \AA^{6}$ \\
\hline I & $\begin{array}{r}0 \\
120\end{array}$ & $\begin{array}{r}-120 \\
0\end{array}$ & $\begin{array}{l}0 \\
0\end{array}$ & $\begin{array}{l}0 \\
0\end{array}$ & $\left.\begin{array}{l}t g^{\prime} \\
g t\end{array}\right\}$ & 73.9 \\
\hline II & $\begin{array}{r}0 \\
105\end{array}$ & $\begin{array}{r}-105 \\
0\end{array}$ & $\begin{array}{l}0 \\
0\end{array}$ & $\begin{array}{l}0 \\
0\end{array}$ & $\left.\begin{array}{l}t g^{\prime} \\
g t\end{array}\right\}$ & 86.0 \\
\hline III & $\begin{array}{r}0 \\
105\end{array}$ & $\begin{array}{r}-105 \\
0\end{array}$ & $\begin{array}{r}0 \\
-10\end{array}$ & $\begin{array}{r}+10 \\
0\end{array}$ & $\left.\begin{array}{l}t g^{\prime} \\
g t\end{array}\right\}$ & 88.0 \\
\hline IV & $\begin{array}{r}0 \\
105\end{array}$ & $\begin{array}{r}-105 \\
0\end{array}$ & $\begin{array}{l}+10 \\
-10\end{array}$ & $\begin{array}{l}+10 \\
-10\end{array}$ & $\left.\begin{array}{l}t^{\prime} \\
g t\end{array}\right\}$ & 113.5 \\
\hline V & $\begin{array}{r}0 \\
105\end{array}$ & $\begin{array}{r}-105 \\
0\end{array}$ & $\begin{array}{l}+20 \\
-20\end{array}$ & $\begin{array}{l}+20 \\
-20\end{array}$ & $\left.\begin{array}{l}\operatorname{tg}^{\prime} \\
g t\end{array}\right\}$ & $\begin{array}{c}137.3 \\
\left(\gamma_{\mathrm{exp}}^{2}=137.5\right)\end{array}$ \\
\hline
\end{tabular}




\section{G. Fourche and B. Lemaire}

the Pauling electronic interactions between adjacent atoms, and a dipolar term for the phenyl rings. Our experimental results are not in contradiction with such a model. However in order to interpret more closely the experimental values, we have been led to introduce a second rotation of identical sign and amplitude on the other phenyl group. Thus in model IV we observe that the effect on $\left\langle r^{2}\right\rangle$ of an additional rotation of $10^{\circ}$ is an increase of about $30 \%$, which is more significant than the previous results. This is easily explained by the fact that in this arrangement the phenyl rings take a disposition markedly more parallel that before. Finally, the comparison of experimental $\left(137.5 \AA^{6}\right)$ and calculated molecular optical anisotropy for meso-DPP allowed us to determine, with a precision of about $\pm 2^{\circ}$, an amplitude of rotation of $20^{\circ}$ for the phenyl rings as indicated in model $\mathrm{V}$. Let us point out in concluding this study that it would have been possible by giving a zero value to the angles $\chi$, to find again the experimental optical anisotropy simply be modifying the internal rotation angles relative to positions $g$ and $g^{\prime}$. However the obtained values, of the order of $60^{\circ}$, are much too small to be reasonably admitted. On the contrary we have verified that for $\chi=19-20^{\circ}$ the arrangement of the rings was still sterically compatible with that of the neighboring atoms. Thus the steric overlap of the van der Waals spheres of the nearest hydrogen atoms (radius $1.2 \AA$ ) is about 0.20 to $0.25 \AA$.

\section{Racemic-DPP}

On the basis of the observations already presented about extremely low values for the statistical weights $\omega, \omega^{\prime}$, and $\omega^{\prime \prime}$, one can see here again that for a dyad dl only two conformations, $t t$ and $g g$, are allowed in the racemic-DPP. Their statistical weights are respectively $\eta^{2}$ and 1. In the $t t$ conformation two phenyl groups are in gauche position with respect to a $\mathrm{CH}$ separated from these group by three skeletal bonds. If we admit, as indicated ${ }^{11}$ by the calculation, that the internal rotation minima in this molecule are very nearly identical to these of meso-DPP, that is $0^{\circ}$ for trans forms $t$ and $+105^{\circ}$ for gauche forms $g$, the average molecular optical anisotropy of this molecule is only dependent on $\eta$ and $\chi$. The examination of molec- ular models readily shows that in the $t t$ form the phenyl groups are parallel for $\chi=0$, and on the contrary in the $g g$ form the planes of these phenyls, always for $\chi=0$, become approximately perpendicular. The previous discussion with regard to meso-DPP allows us to predict that the main contribution to the optical anisotropy of racemic-DPP should arise from its planar $t t$ conformation. Consequently, it would be useful to know precisely the relative position of the phenyl rings in this conformation. However, we can see qualitatively that a slight alteration in the position of the rings, up to about 10 or $15^{\circ}$, does not greatly modify their initial parallelism. It follows that $\left\langle\gamma^{2}\right\rangle$ should not be very sensitive to the angle $\chi$. This is well indicated in Table II.

The observed derivations range from 2.8 to $6 \%$ and thus hardly go beyond the experimental accuracy. (It is important to note that in the case of meso-DPP a displacement of only $2^{\circ}$ of the angles $\chi$ gives rise to the same amount of relative variation of $\left\langle\gamma^{2}\right\rangle$.)

Under these conditions, it is imposible to determine precisely the respective orientation of the phenyl groups of each of the $t t$ and $g g$ forms in the racemic-DPP; whence we have admitted the possibility* of symmetrical torsional oscillations of these groups about their equilibrium state $\chi=0$, with an approximate amplitude of

Table II. Calculated values of $\left\langle\gamma^{2}\right\rangle$ of racemic-DPP for various angles $\chi^{\mathrm{a}}$

\begin{tabular}{rrrl}
\multicolumn{1}{c}{$\chi_{1}$} & $\chi_{2}$ & $\left\langle\gamma^{2}\right\rangle, \AA^{6}$ & Deviation, \% \\
\hline 0 & 0 & 133.69 & 0 \\
+15 & +15 & 126.80 & 5 \\
+15 & -15 & 125.44 & 6 \\
+15 & 0 & 129.92 & 2.8 \\
0 & -15 & 127.77 & 4.4 \\
\hline
\end{tabular}

a $\varphi, 0, \pm 120^{\circ}$; tetrahedral valence angles, $\eta, 1.50$; $\gamma_{\varphi}, 6.97 \AA^{3} ; \gamma_{\mathrm{CC}}, 1.31 \AA^{3} ; \gamma_{\mathrm{CH}}, 0.22 \AA^{3}$.

* In the case of meso-DPP we have seen that we were unable to interpret the experiment by admitting such fluctuations because they lowered $\left\langle\gamma^{2}\right\rangle$ instead of increasing it. However, even if we admit the fluctuation around $\chi=19-20^{\circ}$ their amplitude must be very weak to be compatible with steric overlaps. Thus we have neglected them. 
Table III. Conformational parameters of racemic-DPP

\begin{tabular}{rrcc}
\hline$\varphi_{1}$ & $\varphi_{2}$ & $\chi_{1}, \chi_{2}$ & $\begin{array}{c}\text { Confor- } \\
\text { mation }\end{array}$ \\
\hline 0 & 0 & $\begin{array}{c}\text { Symmetrical torsional } \\
\text { oscillations, } \pm 15^{\circ}\end{array}$ & $\mathrm{tt}$ \\
105 & 105 & $\begin{array}{c}\text { about } \chi=0^{\circ} . \\
\mathrm{gg}\end{array}$ \\
\hline
\end{tabular}

$\pm 15^{\circ}$, as comparable to the experimental precision.

The comparison of the experimental molecular optical anisotropy of racemic-DPP, $131.1 \AA^{6}$ (average of the values observed in cyclohexane and 1,1,3-trimethylcyclohexane), with that calculated by eq 9 according to the choice of the parameters in Table III, allowed us to determine $\eta$ at $25^{\circ} \mathrm{C}$. The obtained statistical weight $\eta=$ $1.48 \pm 0.06$ is in excellent agreement with the result in the literatures. ${ }^{13,15,19}$

A value of $\eta$ greater than unity reveals interactions favoring the trans rotational state in which $\mathrm{C}_{6} \mathrm{H}_{5}$ rather than $\mathrm{CH}_{2}$ is gauche with respect to $\mathrm{CH}$. In other words it also means that the $\operatorname{tg}^{\prime}$ or $g t$ conformation of weight $\eta$ in meso pentane is more favored than that of corresponding type $g g$ or $g^{\prime} g^{\prime}$ in normal alkanes. Indeed, the total statistical weight of the $t^{\prime}{ }^{\prime}$ or $g t$ conformation calculated relatively to a reference trans state, without normalizing to the gauche state of weight $^{6} \sigma$, is $\tau \sigma=\eta \sigma^{2}=1.48(0.363)^{2}=0.194$. The equivalent conformation in normal alkanes is $g g$ or $g^{\prime} g^{\prime}$ and its statistical weight at $25^{\circ} \mathrm{C}$ is $\sigma^{2}=0.131$. Finally this value of $\eta$ exceeding unity also indicate that the extended $t t$ conformation in the racemic-DPP will be clearly preferred to the $g g$ conformation.

Thus, after determining by means of the dimer molecule the ensemble of conformational parameters governing the short distance interactions in PS chains, we are now going to try to account for the experimental optical anisotropies of isotactic and atactic polymers.

\section{ISOTACTIC POLYSTYRENE}

All the calculations have been carried out by using $\eta=1.48, \tau=\eta \sigma=1.48 \times 0.363=0.537$, and $\omega=\omega^{\prime}=\omega^{\prime \prime}=0$. We have observed that if a small (about 0.04-0.05) but nonzero value is attributed to the statistical weights of type $\omega$, it is not possible to account for the experimental optical anisotropies of both isotactic and atactic PS. In particular, we obtain a very small value for $\left(\left\langle\gamma^{2}\right\rangle / x\right)_{\infty}$ of isotactic PS, and in addition the variations of this ratio with $W_{\text {iso }}$ (one can usefully refer to Figure 5 of ref 8 ) are in contradiction with the experiment. Indeed, it is found that $\left(\left\langle\gamma^{2}\right\rangle \mid x\right)_{\infty}$ decreases instead of increasing from the atactic to the isotactic chain.

The bond optical anisotropies and the valence angle used are those previously indicated on p 479. Also the angles $\varphi$ and $\chi$ chosen for mesoand racemic-DPP are those corresponding to model II (Table I) and those reported in Table III, respectively.

Taking into account the deductions already made about 2,4-diphenylpentane we see that the preferred conformations in solution for isotactic polymer should consist, through the $(g t) \rightarrow\left(t g^{\prime}\right)$ transition, of a succession of right-handed $(g t)(g t)(g t) \cdots$ and left-handed $\left(t g^{\prime}\right)\left(t g^{\prime}\right)\left(t g^{\prime}\right) \cdots$ helices, that is $(g t)(g t) \cdots(g t)\left(\operatorname{tg}^{\prime}\right) \cdots\left(\operatorname{tg}^{\prime}\right)\left(\operatorname{tg}^{\prime}\right)$. Let us remark that the reverse transition $\left(\operatorname{tg}^{\prime}\right) \rightarrow$ $(g t)$ is strongly disfavored for it involves a statistical weight $\omega \cong 0$. The foregoing conclusions are for an isotactic ddd... chain. For an 111 ... chain $g$ must be replaced by $g^{\prime}$ and vice versa. On the basis of the results mentioned above, the calculations indicate that the $\left\langle\gamma^{2}\right\rangle / x$ ratios diverge with $x$, that is, they increase very rapidly without any possible limit. Therefore it does not seem possible with this model to obtain a gaussian behavior of the chain as the experiment tends to show. Nevertheless, in order to examine more thoroughly the possibility of such a rigid helical structure of the chain, we have carried out, with this model and according to eq 5 of part I, a theoretical calculation of the weight average value of $\left\langle\gamma^{2}\right\rangle / x$ by means of the Kawahara and Okada's ${ }^{20}$ ponderal distribution of molecular weights. The isotactic PS fractioned by these authors corresponds in fact fairly well to that used here, since it possesses a weight and number-average molecular weights equal to $\bar{M}_{w} \cong 700000$ and $\bar{M}_{n}=150000$, respectively, that is, a polydispersity $\bar{M}_{w} / \bar{M}_{n} \cong 4.6$. Therefore for each molecular weight of the various fractions, we have made 


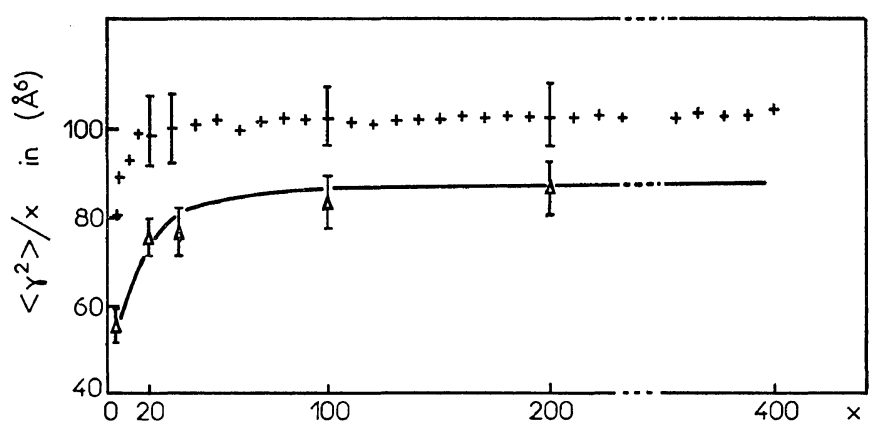

Figure 5. Each point represents the average of the results obtained with 25 Monte Carlo chains. Sampling error is indicated by vertical bars: +++ , calculated values with $W_{\text {iso }}=0.50 ; \triangle$, average of the experimental values obtained in the different solvents.

the calculation of $\left\langle\gamma^{2}\right\rangle / x$ and weighted this result by the weight percentage of the corresponding fraction. The result it as follows:

$$
\left\langle r^{2} / x\right\rangle_{\text {calc }}=110197\left(\AA^{6}\right)
$$

This is a value approximately 100 times as large as that experimentally found:

$$
\left\langle\gamma^{2} \mid x\right\rangle_{\infty, \exp } \cong 967 \pm 100\left(\AA^{6}\right)
$$

It thus seems unlikely that the knowledge of the exact distribution of molecular weights of polymers studied here would markedly improve the result. Consequently we were led to admit, together with some other authors ${ }^{9,21}$, the presence in the chain of occasional structural units disposed in a nonisotactic arrangement. These atypical structures probably have a syndiotactic nature. Therefore we have investigated the dependence of $\left\langle\gamma^{2}\right\rangle / x$ on the probability $W_{\text {syn }}=$ $1-W_{\text {iso }}$ to realize chains having some syndiotactic units ramdomly interspersed among isotactic units. In so doing we have generated 25 Monte Carlo chains composed of 400 monomer units for each value of $W_{\mathrm{s} \text { so }}$ and extrapolated the results to obtain the limiting value of $\left\langle\gamma^{2}\right\rangle / x$. We have found $\left(\left\langle\gamma^{2}\right\rangle / x\right)_{\infty, \mathrm{calc}} \cong 950 \AA^{6}$ for $W_{\text {iso }}=$ $0.96-0.97$, i.e., the presence of about 3 or $4 \%$ of heterotactic units. This $\%$ was sufficient to reconcile theory and experiment. This corresponds to an average length (see appendix) of isotactic sequences of 14 to 18 monomer units, that is about 5 to 6 helix turns of a type close* to $3_{1}$. This estimate is in close agreement with those reported in the literature ${ }^{10,22,23}$ (10 to 15 monomer units) for isotactic chains crystallizable in the form of a $3_{1}$-helix. It is also in satisfactory accord with NMR studies made by Stehling ${ }^{21}$ on isotactic polypropylene. Stehling has estimated the upper permissible limit of atactic units in this polymer to be $5 \%$.

Finally it remains to consider how to reconcile this small proportion of heterotactic units with the highly crystalline properties of isotactic polymers. With regard to this topic we can remark, as shown by Flory, et al., ${ }^{24}$ that to be tolerable in the lattice the syndiotactic units must be incorporated in the chain in such a way that the successive helical segments have parallel (but not colinear) axes. This condition is of course not necessary in solutions where the following possible conformation can be found.

$$
(g t)(g t) \cdots(g t)\left(t g^{\prime}\right)\left(t g^{\prime}\right) \cdots(t t)\left(g^{\prime} t\right)\left(g^{\prime} t\right) \cdots
$$

where $(t t)$ denotes the syndiotactic pair.

\section{ATACTIC POLYSTYRENE}

We have used here the same choice of parameters as for isotactic PS and interpreted the experimental values by varying the proportion of syndiotactic units in the polymer. Each value of $\left\langle\gamma^{2}\right\rangle / x$ has been calculated as the average of the results obtained on 25 Monte Carlo

* The $3_{1}$-helix corresponds in fact to internal rotation angles located at $\varphi=0, \pm 120^{\circ}$ very precisely, which is not the case in this study. 
chains. The best agreement which our experimental results has been realized (see Figure 5) for a probability $W_{\text {iso }}=0.45-0.55$, i.e., 45 to $55 \%$ of heterotactic units. The limiting value, $\left(\left\langle\gamma^{2}\right\rangle / x\right)_{\infty, \text { calc }} \cong 100 \pm 9 \AA^{6}$, obtained by extrapolation from chains of 400 repeating units is however $10 \%$ higher than that experimentally found,

$$
\left(\left\langle r^{2} / x\right\rangle\right)_{\infty, \exp } \cong 89 \pm 5 \AA^{6} .
$$

The same is true for other theoretical points of the curve as seen in Figure 5. However, it must be noticed that if we draw the experimental error on this figure we see that the displacement between theoretical and experimental curves hardly exceed the experimental error, except for low degrees of polymerization where the deviations are more important. In this last case we can notice that the calculation of $\left\langle\gamma^{2}\right\rangle / x$ by a Monte Carlo method becomes somewhat less accurate than that for long chains. Nevertheless the sampling error cannot totally explain the deviations observed.

It could be possible that the discrepancy observed between theory and experiment on the ensemble of the curve arises from a slight deviation of the conformational parameters of the dimer when it is included in the chain, as suggested earlier ${ }^{11}$. Thus noticeably weaker orientational correlations between phenyl rings might perhaps allows to explain the lower values experimentally obtained. In accordance with this hypothesis together with the assumption that the ethylbenzene molecule can be pictured as an isolated monomer unit we have noted:

$$
\left\langle\gamma^{2}\right\rangle_{\text {fraction } 560} \cong x\left\langle\gamma^{2}\right\rangle_{\text {ethylbenzene }}
$$

We have $\left\langle\gamma^{2}\right\rangle_{\text {ethylbenzene }}=60.7 \AA^{6}$ and $\left(\left\langle\gamma^{2}\right\rangle /\right.$ $x)_{\text {fraction } 560} \cong 58.7 \AA^{6}$ from measurements carried out in cyclohexane. This result indicates an important decrease in the internal orientational correlations between phenyl rings in this sample of very low molecular weight. For other fractions, Table I (part I) shows that it is not possible to recover the experimental optical anisotropy simply by multiplying $x$ times the optical anisotropy of the isolated element.

We have assumed in the course of our interpretation that the distribution of syndiotactic units along the chain was perfectly Bernoullian. In fact it has been shown that according to the operative conditions used, a polymer might or might not obey such a statistic. Thus Bovey ${ }^{25}$ has found that poly(methyl methacrylate) obtained by anionic polymerization did not obey a Bernoullian statistic. Presently most of the atactic PS studied were synthetized by anionic polymerization techniques ${ }^{26}$. Therefore the distribution process of heterotactic sequences in the PS we have used could be not exactly Bernoullian, and this would introduce an additional discrepancy into the experimental interpretation. However, the average length of the isotactic sequences (see appendix) of 2 or 3 monomer units, which is about one turn of a $3_{1}$-helix, found with the Bernoullian model appears to be reasonable and in close agreement with recent observations, ${ }^{23}$ according to which helical segments would exist in atactic PS in solution. Another indirect confirmation of our results seems to be given by an X-ray study by $\mathrm{Killian}^{27}$ on solid atactic PS. Indeed, this author has interpreted these measurements by assuming the existence in the solid polymer of isotactic sequences having an average length equivalent to 2 or 3 turns of a $3_{1}$-helix.

The conformation of atactic PS as a whole, considered in terms of a local structure corresponding to the dimer molecule, is then of the same nature as for isotactic PS, but the ordered sequences are much shorter than in the stereoregular polymer.

\section{DISCUSSION}

At this point the following model can be proposed for the conformation of PS in solution. The isotactic PS contains long ordered sequences composed of very regular chain separated by heterotactic regions randomly distributed along the molecule. It has been supposed that in rigid sequences the aliphatic backbone and the phenyl rings take a conformational arrangement practically identical to that observed in crystalline isotactic PS.

The atactic PS also contains helical sequences but these are shorter than for the isotactic polymer. The difference in conformation from the stereoregular polymer is not therefore a difference in nature but rather in degree of tacticity.

In order to examine the influence of stereoregularity on molecular optical anisotropy we 


\section{G. Fourche and B. Lemaire}

have drawn (Figure 6) the curve $\left(\left\langle\gamma^{2}\right\rangle / x\right)_{\infty} v s$. $W_{\text {iso }}$ with our choice of parameters. Each point of the curve represents the average for 25 Monte Carlo chains consisting of 400 repeating units, which is a sufficient number to reach asymptotic behavior. We observe an extremely sharp decrease of $\left(\left\langle\gamma^{2}\right\rangle / x\right)_{\infty}$ when $W_{\text {iso }}$ increases from 0 (syndiotactic) to approximately 0.20 . The variation of the optical anisotropy becomes very much weaker in the range $0.20 \leq W_{\text {iso }} \leq 0.40$ and in the region between 0.45 to 0.55 it is practically independent of the degree of tacticity. This is quite understandable in view of the fact that in this domain of stereoregularity the internal orientational correlations between successive units of the chain are minima.

On the contrary when $W_{\text {iso }}$ rises again from

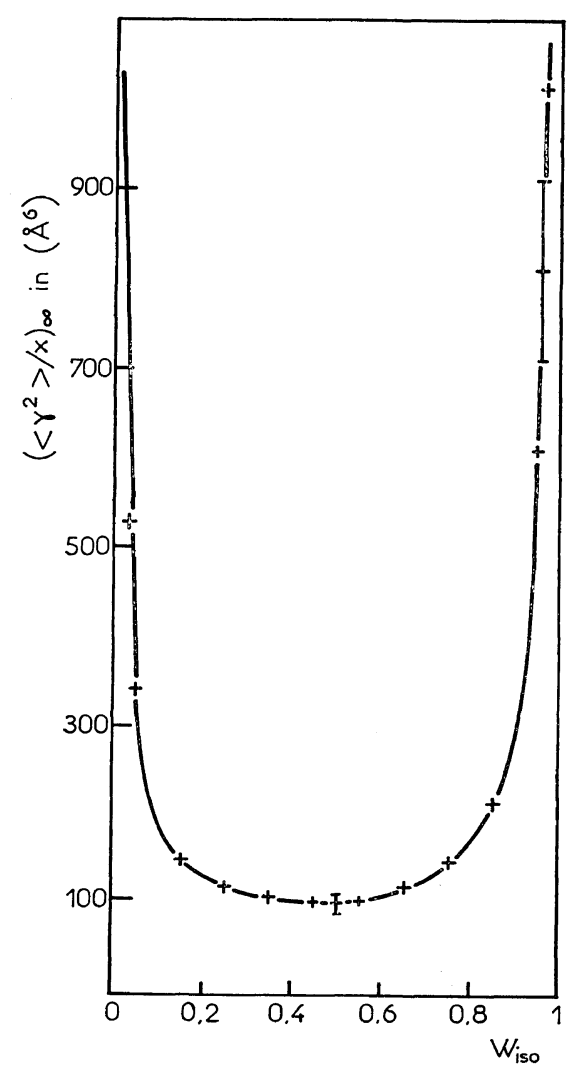

Figure 6. Values of $\left(\left\langle\gamma^{2}\right\rangle / x\right)_{\infty}$ calculated by extrapolation from chains of 400 repeating units. Each point is the average of results obtained for 25 Monte Carlo chains. Sampling error is indicated by vertical bars.
0.5 (atactic) to 1 , (isotactic) $\left(\left\langle\gamma^{2}\right\rangle / x\right)_{\infty}$ also increases very rapidly with the reappearance of molecular regularity. The examination of Figure 6 , where the sampling error has been indicated in the isotactic and atactic regions (the only ones studied here), shows that the measure of molecular optical anisotropy should be a useful method for the determination, with satisfactory precision, ${ }^{*}$ of the degree of stereoregularity of PS chains. Indeed when $W_{\text {iso }}$ rises from 0.5 to 0.95 the ratio $\left(\left\langle\gamma^{2}\right\rangle \mid x\right)_{\infty}$ increases from 100 to $600\left(\AA^{6}\right)$ (Figure 6) but the characteristic ratio $\left(\left\langle R^{2}\right\rangle_{0} / n l^{2}\right)_{\infty}$ calculated $^{9}$ for polypropylene chains (the structural parameters together with the various statistical weights of PP chains ${ }^{9,24}$ being practically those of PS) only increases under the same conditions from 8 to 28 . Thus the stereoregularity has twice as important an effect on the optical anisotropy as on the chain extension. Qualitatively, this explains well the lack of sensitivity of the characteristic ratio to the stereoregularity. Quantitatively, however, an important discrepancy remains between theoretical and experimental predictions if it is assumed that the measures of chain dimensions have been carried out on samples of comparable degrees tacticity.

To summarize the above discussion: we have quantitatively verified, by means of a method sensitive exclusively to short distance interactions, the hypothesis according to which many helical rigid sequences must exist in isotactic PS and to a lesser extent in atactic PS. This study also permitted us, in the case of the dimer molecule, to establish the arrangement of the phenyl rings around the aliphatic skeleton. It is useful to know this orientation in order to be able to interpret theoretically the hyperchromic and hypochromic phenomena as well as the fluorescence of these molecule. Finally, the interpretation of these results underlines the prominent role played by classical van der Waals forces, which alone can create suitable conditions for short distance intramolecular order in the molecule.

* Let us remark that the limit of the method is more on theoretical side than on the experimental one because the precision of $\left\langle\gamma^{2}\right\rangle$ measurements is notably better than that obtained from calculation. 


\section{GENERAL CONCLUSION}

In an overall point of view, we may conclude that the present work and Part I of this series have pointed out the high sensitivity of molecular optical anisotropy to internal orientational correlations in the chain molecules. These results also demonstrate that in general one should be able to determine with sufficient accuracy and in a manner free from other effects (i.e., uneffected by other additional phenomena, such as excluded volume and interference, when the molecular weight increases) the optical anisotropy of low molecular weight compounds as well as that of long chains.
In addition, a consistent interpretation of a number of experimental optical anisotropies of oligomers together with those of polymers studied in several solvents was given, in close agreement with results obtained elsewhere.

\section{APPENDIX}

Let us examine the calculation, for very long chains, of the average length $\langle L\rangle$ of isotactic sequences ddd $\ldots$ or $111 \ldots$ taking into account the possibility of reversing the helix screw sense. From the following set of conformations with $x$ repeating unit in isotactic arrangement,

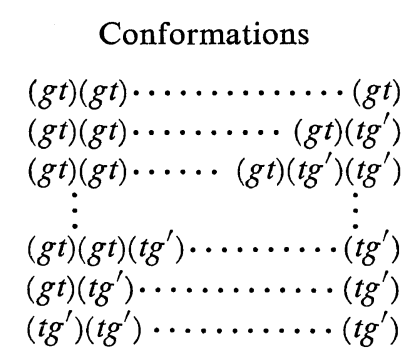

Total number of repeating units in helical sequence

$$
\begin{gathered}
x \\
x-1 \\
x \\
\vdots \\
x \\
x-1 \\
x
\end{gathered}
$$

we obtain the length $L_{x}$ of an isotactic sequence involved in a chain of $x$ units.

$$
L_{x}=\frac{\text { Total number of repeating units in helical sequences }}{\text { Number of helical sequences }}=\frac{(x-1)(x+2)}{2(x-1)}=\frac{x+2}{2}
$$

Under these conditions the average length of an isotactic sequence of the chain is

$$
\langle L\rangle=\frac{\sum_{x=2}^{\infty} W_{\mathrm{iso}}^{x-1} \cdot W_{\mathrm{syn}} \cdot L_{x}}{\sum_{x=2}^{\infty} W_{\mathrm{i} \text { so }}^{x-1} \cdot W_{\mathrm{syn}}}=3 / 2+1 /\left(2 W_{\mathrm{syn}}\right)
$$

Let us remark that for very long and predominantly isotactic chains, $W_{\text {syn }} \ll 1$ and consequently $\langle L\rangle \cong 1 /\left(2 W_{\mathrm{syn}}\right)$.

\section{REFERENCES}

1. V. N. Tsvetkov, J. Polym. Sci., 57, 727 (1962); V. N. Tsvetkov, S. Ya Magarik, N. N. Boitsova, and M. G. Okuneva, ibid., 54, 635 (1961).

2. H. Utiyama, J. Phys. Chem., 69, $\mathrm{n}^{\circ} 12,4138$ (1965); H. Utiyama and M. Kurata, Bull. Inst. Chem. Res., Kyoto Univ., 42, 128 (1964).

3. G. Fourche, J. Chim. Phys., 65, 1500 (1968), ibid., 66, 320 (1969).
4. R. L. Jernigan and P. J. Flory, J. Chem. Phys., 47, 1999 (1967).

5. K. Nagai, J. Chem. Phys., 47, 4690 (1967).

6. G. Fourche and P. Bothorel, J. Chim. Phys., 66, 54 (1969).

7. B. Lemaire and G. Fourche, J. Polym. Sci., Part A-2, 9, 961 (1971).

8. A. E. Tonelli, Y. Abe, and P. J. Flory, Macromolécules, 3 (3), 303 (1970).

9. P. J. Flory, "Statistical Mechanics of Chain Molecules", Interscience Publishers Inc., New York, N. Y., 1969.

10. T. M. Birshtein and O.B. Ptitsyn, "Conformations of Macromolecules", Interscience Publishers Inc., New York, N. Y., 1966.

11. S. Gorin and L. Monnerie, J. Chim. Phys., 67, 869 (1970).

12. C. Such, C. Clement, and P. Bothorel, C.R. Acad. Sci., 271, 228 (1970).

13. F. A. Bovey, F. P. Hood, E. W. Anderson, and L. C. Snyder, J. Chem. Phys., 42, 3900 (1965).

14. F. Heatley and F. A. Bovey, Macromolécules, 


\section{G. Fourche and B. Lemaire}

1, 4, 301 (1968).

15. A. D. Williams and P. J. Flory, J. Amer. Chem. Soc., 91, 3111 (1969).

16. A. Unanue, Thèse Bordeaux, 1965.

17. Y. Abe, A. E. Tonelli, and P. J. Flory, Macromolécules, 3, (3), 294 (1970).

18. B. Lemaire, G. Fourche, and P. Bothorel, to be published.

19. D. Lim, M. Kolinsky, J. Petranek, D. Doskocilova, and B. Schneider, J. Polym. Sci., Part B, 4, 645 (1966); H. Pivcova, M. Kolinsky, D. Lim, and B. Schneider, Preprint Bruxelles, 1967.

20. K. Kawahara and R. Okada, J. Polym. Sci. 56, 163 (1962).
21. F. C. Stehling, J. Polym. Sci. Part A-2, 1815 (1964).

22. P. J. Flory, J. E. Mark, and A. AвE, Polymer Letters, 3, 973 (1965).

23. C. Reiss, J. Chim. Phys., 63, 1299 (1966).

24. P. J. Flory, J. E. Mark, and A. Abe, J. Amer. Chem. Soc., 88, 639 (1966).

25. F. A. Bovey, Accounts Chem. Res., $\mathrm{n}^{\circ} 6,175$ (1968).

26. T. Altares, Jr, D. P. Wyman, and V. R. Allen, J. Polym. Sci., Part A, 4533 (1964).

27. H. G. Killian and K. Boueke, J. Polym. Sci., 58, 311 (1962). 